\title{
Daidzein ameliorates spinal cord ischemia/reperfusion injury-induced neurological function deficits in Sprague-Dawley rats through PI3K/Akt signaling pathway
}

\author{
FAN ZHANG* , NENG RU* ${ }^{*}$ ZHENG-HUI SHANG*, JIAN-FENG CHEN, CHAO YAN, YUN LI and JIE LIANG
}

Department of Orthopedics, The First People's Hospital of Yichang,

China Three Gorges University, Yichang, Hubei 443000, P.R. China

Received August 2, 2016; Accepted May 5, 2017

DOI: $10.3892 /$ etm.2017.5166

\begin{abstract}
Daidzein (DZ) has a broad spectrum of biological activities, including antioxidant, anti-inflammatory and anticancer as well as cardio- and hepatoprotective properties. The present study was designed to elucidate the in-depth mechanism underlying the neuroprotective efficacy of DZ against spinal cord ischemic/reperfusion injury (SCII) in a rat model by comparison with the standard neuroprotective agent methylprednisolone (MP). A total of 48 rats were divided into four groups of twelve rats in each $(\mathrm{n}=12)$. In sham-operated group (Control) group, rats received only saline (Fogarty catheter was inserted without balloon inflation), whereas rats in the SCII induction group (SCII) were subjected to SCII insult by insertion of a Fogarty balloon catheter, which was inflated in the descending thoracic aorta to cause an occlusion. A proportion of rats was treated with DZ $(20 \mathrm{mg} / \mathrm{kg}$; DZ+SCII group) or MP (50 mg/kg; MP+SCII group) for seven days prior to and after SCII. The locomotor function (neurological activity) and antioxidant levels (superoxide dismutase and catalase) levels were significantly improved upon treatment with DZ and MP in comparison with those in the SCII group. A concomitant decline in edema, inflammatory markers (myeloperoxidase, tumor necrosis factor- $\alpha$ and nuclear factor $\kappa \mathrm{B}$ p65), the apoptotic marker caspase-3 and the number of cells with terminal deoxynucleotidyl transferase-mediated dUTP nick-end labeling was also observed in the DZ and MP groups. The protein levels of phosphoinositide-3 kinase (PI3K), the phosphorylated Akt/Akt ratio and B-cell lymphoma 2 (Bcl-2) were substantially downregulated, while
\end{abstract}

Correspondence to: Dr Jie Liang, Department of Orthopedics, The First People's Hospital of Yichang, China Three Gorges University, 2 Jiefang Road, Xiling, Yichang, Hubei 443000, P.R. China

E-mail: liangjielj@hotmail.com

${ }^{*}$ Contributed equally

Key words: spinal cord ischemia reperfusion, daidzein, methylprednisolone, edema, inflammatory markers, apoptosis
Bcl-2-associated X protein levels were upregulated SCII insult group, which was inhibited by treatment with DZ. To conclude, pre-treatment with DZ significantly improved the neurological function by upregulating PI3K/Akt signaling and thereby considerably attenuating the inflammatory response and apoptosis, thus maintaining the neuronal count in an SCII-induced rat model.

\section{Introduction}

Spinal cord injury (SCI) is a devastating complication of thoracic or thoracoabdominal aortic reconstruction/repair that leads to disabilities, particularly paraplegia and quadriplegia. It has been estimated that $15-80 \%$ of cases of acute traumatic neuronal damage (primary spinal injury) or aortic surgery, which are primary spinal injury, may result in paraplegia or quadriplegia $(1,2)$. Paraplegia and quadriplegia caused by traumatic primary spinal cord injury are irreversible and hence, several studies have focused on treating secondary spinal injury due to ischemia/reperfusion, hypoxia and glutamate excitotoxicity that are reversible $(3,4)$. The present study used a rat model of spinal cord ischemia/reperfusion injury (SCII) in order to elucidate the pathophysiology of secondary SCII, which has remained to be fully elucidated (5). However, various studies have proposed that inflammation, apoptosis and oxidative stress are the major contributors to the development of SCII and associated conditions $(6,7)$. Hence, a drug that subsides inflammation and apoptosis along with an improved anti-oxidant capacity would be the best option for treating SCII conditions.

A large amount of evidence indicated that soy extracts possess neuroprotective activity against brain injury/trauma owing to the estrogenic effects of phytoestrogens (isoflavones), such as daidzein (DZ; 4',7-dihydroxyisoflavone) and genistein $(8,9)$. DZ is found in soybeans, soy-based products (tofu), red clover (Trifolium pratense) as well as in Chinese herbs such as Kwao Krua (Pueraria mirifica) and Kudzu (Pueraria lobate) at higher concentrations (10). DZ may be metabolized by intestinal bacteria to S-equol, which has a broad spectrum of health-promoting benefits, including antioxidant, anti-inflammatory, anticancer, anti-obesity as well as cardio- and hepatoprotective properties (10-12). 
All these beneficial effects of DZ are directly linked to its structural similarity to estrogen (17 $\beta$-estradiol). Furthermore, DZ/S-equol have been found to act as agonists of G-protein-coupled estrogen receptor, thus regulating various signaling pathways (13). In addition, DZ and genistein also influence the cell survival rate by desactivating the caspase cascade by positively regulating the phosphoinositide-3 kinase (PI3K) signaling pathway $(14,15)$. Methylprednisolone (MP) is a well-known neuroprotective agent that possesses antioxidant, anti-inflammatory and anti-apoptotic properties (16), and was hence used as a standard in the present study.

Apoptosis (programmed cell death) is the major contributor to neuronal death, which is activated by a caspase-dependent and -independent pathway (17). In most tissues, cell survival and proliferation are positively regulated (coordinated) by the PI3K/Akt signaling pathway via modulating several downstream molecules, such as mammalian target of rapamycin (mTOR), nuclear factor erythroid 2 -related factor (Nrf2) and glycogen synthase kinase-3 $\beta$ (GSK-3 $\beta)(18,19)$. Furthermore, apoptosis is one of the crucial factors for neuronal loss, particularly that associated with SCII (17). Hence, the present study was designed to assess whether DZ exerts its neuroprotective effect by positively upregulating the PI3K/Akt pathway to inhibit neuronal apoptosis and reduce damage in the rat model of SCII.

\section{Materials and methods}

Drugs and reagents. DZ, MP, SDS and Tris buffer were procured from Sigma-Aldrich (Merck KGaA, Darmstadt, Germany). Isoflurane, ketamine, physiological saline $(0.89 \%)$, formaldehyde, DAPI stain, PBS and xylene were purchased from Beijing Zhongshan Goldenbridge Biotechnology Co. Ltd. (Beijing, China). All of the other chemicals were of analytical grade.

Experimental rats. Male Sprague Dawley (SD) rats $(\mathrm{n}=48$; weight, 320-350 g; age, 2 months) were procured from The First People's Hospital of Yichang Animal House (Hubei, China). Rats were maintained in an animal facility center in a steel cage, (4 rats in each cage), under a 12-h light/dark cycle with ad libitum access to food and water. The study protocol was approved by the Institutional Review Board of China Three Gorges University (RHTGU.930482), and was according to the National Institutes of Health Guide for the Care and Use of Laboratory Animals.

SCI insult. SCI injury was performed according to the method used by Hwang et al (20) with a slight modification. In brief, all rats were anesthetized with ketamine $(50 \mathrm{mg} / \mathrm{kg})$ intraperitoneally (i.p.) under fasting conditions (12 h). Body temperature was stabilized at $37^{\circ} \mathrm{C}$ by warming/heating pads and heating lamps. The rats were placed in a supine position, making sure that neck region was well exposed. The hair on the neck region (carotid-inguinal) was shaved with trimmer/shaver. A polyethylene catheter (PE-50) was inserted into the tail artery to inject heparin as well as to monitor the mean distal arterial pressure (MDAP). A Fogarty balloon catheter (Edwards Life Science, Shanghai,
China) was inserted via the left femoral artery into the proximal descending thoracic aorta $(\sim 11 \mathrm{~cm}$ from the insertion site), and the balloon was inflated to induce spinal cord ischemia. During aortic occlusion, the proximal artery pressure was maintained at $80 \mathrm{mmHg}$ by draining the blood from the carotid artery into the external blood reservoir filled with heparinized saline. Aortic occlusion (ischemia) was confirmed by immediate loss of pulse with decreased MDAP. The balloon was deflated after $25 \mathrm{~min}$ of ischemia to initiate reperfusion by restoring blood flow. The catheters were then slowly withdrawn, and the rats were allowed to recover from anesthesia. All of these procedures, except for aortic occlusion, were performed in sham-operated control rats, which served as negative controls. Mean arterial pressure, body temperature and heart beat (vitals) were continuously monitored throughout the study.

Experimental design. After one week of assimilation, the $48 \mathrm{SD}$ rats were divided into four treatment groups $(\mathrm{n}=12$ per group): Group I, rats receiving saline (sham-operated control) without occlusion; Group II, rats subjected to ischemic insult (SCII); Groups III, and IV; rats treated with DZ $(20 \mathrm{mg} / \mathrm{kg})$ or MP $(50 \mathrm{mg} / \mathrm{kg})$ i.p. in saline for 7 days prior to SCII (pre-treatment) and 7 days after SCII (post-treatment), serving as treatment groups (DZ+SCII; MP+SCII).

Neurological assessment. Locomotor function (hind limb movement) was determined on days 1, 3, 5 and 7 after SCII using the Basso, Beattie and Bresnahan (BBB) motor rating scale. The rating scale comprises 21 items: 0 , no detectable hind limb movement; 1-8, slight or higher movements of hindlimb joints; 9, dorsal stepping; 10-20, progressive improvement of walking ability; and 21, normal movement.

Tissue processing. On day 7, after the last neurological assessment, animals in each group were divided into two sub-groups for biochemical/bolt analyses $(n=6)$ and terminal deoxynucleotidyl transferase-mediated deoxyuridine triphosphate nick-end labeling (TUNEL) assay $(n=6)$. All rats were euthanized on day 7 by i.p. injection of pentobarbital sodium to remove spinal tissue, which was immediately fixed with $4 \%$ paraformaldehyde for $3 \mathrm{~h}$ at $37^{\circ} \mathrm{C}$, followed by dehydration (overnight), embedding in paraffin and slicing at $5 \mu \mathrm{m}$ using a microtome for the TUNEL assay $(\mathrm{n}=6)$. Spinal tissue was homogenized in $10 \%$ (w/v) Tris- $\mathrm{HCl}$ lysis buffer and centrifuged at $2,500 \mathrm{x}$ for $20 \mathrm{~min}$ at $4^{\circ} \mathrm{C}$ for use in biochemical analyses $(n=6)$.

Evaluation of edema (water content). Edema was detected using procedures previously published by Mdzinarishvili et al (21) with certain modifications where the spinal tissue were super infused with $0.5 / \mathrm{min}$ instead of $0.7 / \mathrm{min}$. The wet and dry weights of spinal samples were measured to calculate the water content. Spinal edema was defined as the percentage difference between the two weights.

Anti-oxidant enzymes and myeloperoxidase (MPO) activity assays. Superoxide dismutase (SOD) and catalase (CAT) activity was quantified in spinal tissue (homogenate) using commercial kits (SOD, cat. no. A001; CAT, cat. no. A007; 
Nanjing Jiancheng Bioengineering Institute, Nanjing, China) based on the manufacturer's protocols. One unit (U) of SOD activity is equal to the amount of enzyme that inhibits the auto-oxidation reaction by $50 \%$. One $\mathrm{U}$ of CAT activity in $1 \mathrm{mg}$ of tissue protein was defined as the amount that consumed $1 \mu \mathrm{mol}$ of $\mathrm{H}_{2} \mathrm{O}_{2}$ at $405 \mathrm{~nm}$ during $1 \mathrm{sec}$. MPO activity in spinal homogenate was measured using an MPO assay kit (cat. no. A044; Nanjing Jiancheng Bioengineering Institute) according to the supplier's instructions. One $\mathrm{U}$ of MPO activity was defined as the amount of enzyme degrading $1 \mathrm{mmol}$ peroxidase $/ \mathrm{min}$ at $25^{\circ} \mathrm{C}$ and was expressed as $\mathrm{U} / \mathrm{g}$ of wet tissue.

Inflammatory and apoptotic markers. The nuclear factor (NF)- $\mathrm{kB}$ free $\mathrm{p} 65$ subunit in the nuclear fraction isolated from the spinal tissue using the Nuclear/Cytosolic Fractionation Kit (cat. no. AKR171; Cell Biolabs Inc., San Diego, CA, USA) was evaluated using an ActivELISA kit (cat. no. IMK503; Imgenex; Novus Biologicals, LLC, Littleton, CO, USA). The levels of tumor necrosis factor- $\alpha$ (TNF- $\alpha$ ) and caspase- 3 in spinal tissue (cytosolic fraction) were measured by ELISA using the Quantikine Rat TNF- $\alpha$ and Quantikine Active Caspase-3 kits (TNF- $\alpha$, cat. no. MTA00B; caspase-3, cat. no. KM300/DYC; R\&D Systems Inc., Minneapolis, MN, USA) according to the manufacturer's protocols.

TUNEL assay. Apoptosis (cell death) in spinal tissue was assessed using the In Situ BrdUTP-Red DNA fragmentation kit/TUNEL assay kit from Abcam (cat. no. ab66110; Cambridge, MA, USA), according to manufacturer's instructions. In brief, spinal tissue was fixed in $4 \%$ formaldehyde and washed with PBS for $5 \mathrm{~min}$. The slides were incubated with protease $\mathrm{K}(20 \mu \mathrm{g} / \mathrm{ml})$ and $0.5 \%$ Triton $\mathrm{X}-100$ for $20 \mathrm{~min}$ at $37^{\circ} \mathrm{C}$. Subsequent to washing with PBS, slices were incubated with $50 \mu \mathrm{l}$ TUNEL reaction mixture (red) for $30 \mathrm{~min}$ at $37^{\circ} \mathrm{C}$. Finally, samples were counterstained with DAPI to visualize all nuclei (blue) and apoptotic cells (red/pink) by confocal microscopy. Quantification of positive cells per $\mathrm{mm}^{2}$ was performed at a magnification of $\mathrm{x} 400$ in a double-blinded manner in each section of spinal tissue.

Western blot analysis. For analysis of PI3K, total Akt, phosphorylated (p)-Akt, B-cell lymphoma 2 (Bcl-2) and Bcl-2-associated X protein (Bax) proteins in the spinal tissue, were extracted using RIPA lysis buffer (Sigma-Aldrich; Merck $\mathrm{KGaA}$ ) and the total cellular protein was estimated using a BCA Protein assay reagent kit (BioVision, Inc., Milpitas, CA, USA). Subseuently nuclear/cytosolic proteins were isolated using a Nuclear/Cytosol Extraction kit (cat. no. AKR171; Cell Biolabs Inc., San Diego, CA, USA) based on the supplier's instructions. Equal quantities of protein (50 $\mu \mathrm{g} / \mathrm{lane})$ was separated by $8 \%$ SDS-PAGE and then electrotransferred onto a nitrocellulose membrane by a semi-dry blotting system (GE Healthcare, Little Chalfont, UK). The membrane was blocked with Tris-buffered saline (TBS) containing Tween-20 and 5\% skimmed milk and probed with primary antibody at $4^{\circ} \mathrm{C}$ overnight. The following antibodies were used in the present study: Rabbit polyclonal anti-PI3K (cat. no. sc-67306; 1:1,000 dilution), anti-Akt and p-Akt antibody (cat. nos. sc-5298 and sc-135650; 1:2,000 dilution), mouse

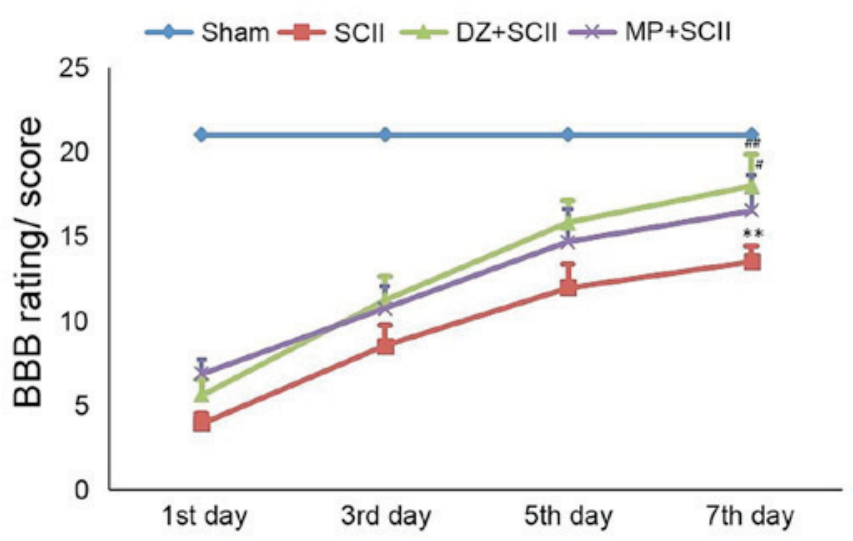

Figure 1. Effect of DZ on locomotor function (BBB rating) in experimental rats. ${ }^{* *} \mathrm{P}<0.01$ vs. sham control group; ${ }^{\# \#} \mathrm{P}<0.01,{ }^{\#} \mathrm{P}<0.05$ vs. SCII group. DZ, daidzein; MP, methylprednisolone; SCII, spinal cord ischemia/reperfusion injury; BBB, Basso, Beattie and Bresnahan.

anti-Bcl-2 antibody and anti-Bax antibody (cat. no. sc-509 and sc-20067; 1:500 dilution) as well as mouse monoclonal anti-rat $\beta$-actin antibody (cat. no. sc-47778; 1:500 dilution) (all from Santa Cruz Biotechnology, Inc., Dallas, TX, USA), which served as an internal control (housekeeping protein). Subsequently, samples were incubated with the secondary antibodies, horseradish peroxidase-conjugated anti-mouse antibody (cat. no. sc-2380/2371; 1:2,000 and 1:5,000 dilution, respectively; Santa Cruz Biotechnology, Inc) in TBS at room temperature for $1 \mathrm{~h}$ and washed with TBS. The bound antibodies were visualized using an enhanced chemiluminescence system (ChemiDoc-17001401; Image Lab-5.2.1; Bio-Rad Laboratories, Inc., Hercules, CA, USA).

Statistical analysis. Values are expressed as the mean \pm standard deviation. SPSS version 23 (International Business Machines, Inc., Armonk, NY, USA) was used for statistical analysis. Variations between groups of experimental rats were evaluated by one-way analysis of variance and the least significant difference was determined using a post-hoc multiple comparison test. $\mathrm{P}<0.05$ was considered to indicate a statistically significant difference.

\section{Results}

Effect of DZ on locomotor function. Fig. 1 portrays the hind limb movement (locomotor function) of sham-operated control and experimental rats. Sham-operated control rats showed normal hind limb movement with an average BBB score of 21 without any neurological deficits. By contrast, SCII rats displayed a concomitant decline in hind limb movement evidenced by paraplegia with the lowest BBB score at all time-points (days $1,3,5$ and $7 ; \mathrm{P}<0.01$ ). Treatment with DZ and MP greatly ameliorated the impairment of hind limb movement by effectively alleviating neuronal damage, which was evidenced by an elevated BBB score compared with that in SCI-insulted rats $(\mathrm{P}<0.01$ and $<0.05)$.

Effect of DZ on spinal edema. The sham-operated rats did not display any edematous changes (Fig. 2A). By contrast, SCI-induced rats had marked edema $(81.35 \% ; \mathrm{P}<0.01$ vs. 
Table I. Effect of DZ on spinal anti-oxidant activities in experimental rats.

\begin{tabular}{lcc}
\hline Group & $\begin{array}{c}\text { SOD } \\
(\mathrm{U} / \mathrm{mg} \text { protein })\end{array}$ & $\begin{array}{c}\text { CAT } \\
(\mathrm{U} / \mathrm{mg} \text { protein })\end{array}$ \\
\hline Sham (control) & $4.03 \pm 0.31$ & $73.53 \pm 9.51$ \\
SCII & $2.89 \pm 0.18^{\mathrm{a}}$ & $46.36 \pm 5.63^{\mathrm{a}}$ \\
DZ+SCII & $3.86 \pm 0.42^{\mathrm{b}}$ & $64.70 \pm 6.84^{\mathrm{b}}$ \\
MP+SCII & $3.48 \pm 0.51^{\mathrm{c}}$ & $59.85 \pm 7.30^{\mathrm{c}}$ \\
\hline
\end{tabular}

${ }^{\mathrm{a}} \mathrm{P}<0.01$ vs. control group; ${ }^{\mathrm{b}} \mathrm{P}<0.01,{ }^{\mathrm{c}} \mathrm{P}<0.05$ vs. SCII group. Values are expressed as the mean \pm standard deviation $(n=12)$. DZ, daidzein; SCII, spinal cord ischemia/reperfusion injury; SOD, superoxide dismutase; CAT, catalase.
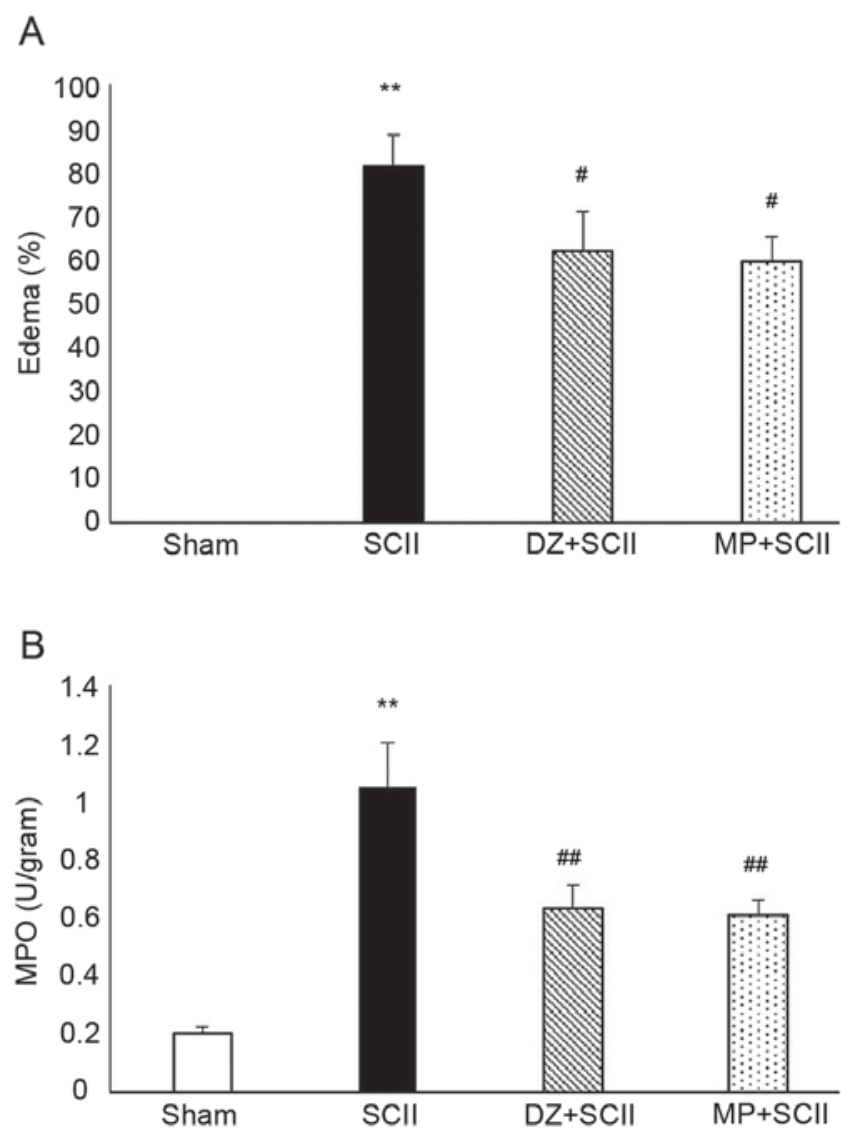

Figure 2. Effect of DZ on (A) spinal edema and (B) MPO levels in experimental rats. ${ }^{* *} \mathrm{P}<0.01$ vs. sham control group; ${ }^{\# /} \mathrm{P}<0.01,{ }^{\#} \mathrm{P}<0.05$ vs. SCII group. DZ, daidzein; MP, methylprednisolone; SCII, spinal cord ischemia/ reperfusion injury; MPO, myeloperoxidase.

Sham group). In comparison with SCI-insulted rats, DZ and MP-treated rats displayed a significant decline in edema levels at 62.12 and $59.56 \%$, respectively $(\mathrm{P}<0.05)$.

Effect of DZ on antioxidant activity. To determine whether DZ alleviates oxidative stress, the levels of the endogenous antioxidants SOD and CAT were determined (Table I). The activities of SOD and CAT in the spinal cords of SCII rats were markedly diminished compared with those in the
Table II. Effect of DZ on spinal inflammatory and apoptotic markers in experimental rats.

\begin{tabular}{lccc}
\hline Group & $\begin{array}{c}\text { TNF- } \alpha \\
\text { (ng/mg ptn) }\end{array}$ & $\begin{array}{c}\text { NF-kB p65 } \\
\text { (pg/mg ptn) }\end{array}$ & $\begin{array}{c}\text { Caspase-3 } \\
\text { (ng/mg ptn) }\end{array}$ \\
\hline Sham (control) & $118.25 \pm 17.23$ & $82.45 \pm 7.25$ & $9.13 \pm 0.51$ \\
SCII & $281.46 \pm 21.68^{\mathrm{a}}$ & $189.57 \pm 17.45^{\mathrm{a}}$ & $35.28 \pm 3.52^{\mathrm{a}}$ \\
DZ+SCII & $137.67 \pm 15.11^{\mathrm{b}}$ & $91.36 \pm 7.24^{\mathrm{b}}$ & $15.63 \pm 1.52^{\mathrm{b}}$ \\
MP+SCI & $132.95 \pm 12.67^{\mathrm{b}}$ & $99.67 \pm 11.46^{\mathrm{b}}$ & $17.57 \pm 1.26^{\mathrm{b}}$ \\
\hline
\end{tabular}

${ }^{\mathrm{a}} \mathrm{P}<0.01$ vs. control group; ${ }^{\mathrm{b}} \mathrm{P}<0.01$ vs. SCII group. Values are expressed as the mean \pm standard deviation $(n=12)$. Ptn, protein; TNF, tumor necrosis factor; NF, nuclear factor; DZ, daidzein; SCII, spinal cord ischemia/reperfusion injury.

sham-operated control rats $(\mathrm{P}<0.05)$. Treatment with DZ at the dosage of $20 \mathrm{mg} / \mathrm{kg}$ significantly abrogated these decreases $(\mathrm{P}<0.01)$, resulting in near-basal antioxidant levels by abolishing the oxidative stress. Administration of MP also substantially improved the levels of SOD and CAT $(\mathrm{P}<0.05)$. However, DZ had a better antioxidant activity when compared with MP.

Effect of DZ on MPO activity. MPO activity was determined to assess the efficacy of DZ on neutrophil activation and infiltration (Fig. 2B). Compared with that in the sham-operated control group, the SCII group displayed increased MPO activity $(\mathrm{P}<0.01)$. In comparison, treatment with $\mathrm{DZ}$ and $\mathrm{MP}$ for 7 days prior to and following SCII significantly restrained MPO activity by inactivating neutrophils $(\mathrm{P}<0.01)$.

Effect of DZ on inflammatory markers. The anti-inflammatory effect of DZ was evaluated by assessing various inflammatory cytokines, including TNF- $\alpha$ in the spinal tissues of the rats and $\mathrm{NF}-\kappa \mathrm{B}$ subunit $\mathrm{p} 65$ in the nuclear fraction of these tissues. The levels of TNF- $\alpha$ and nuclear NF- $\kappa$ B p65 in SCI-insulted rats were significantly increased compared with those in sham-operated rats $(\mathrm{P}<0.01$; Table II). However, DZ and MP substantially suppressed the levels of TNF- $\alpha$ and nuclear translocation of the NF- $\mathrm{B}$ p 65 subunit, thus indicating its anti-inflammatory activity.

Effect of DZ on apoptotic markers. Caspase-3 is a crucial apoptotic protease involved in apoptosis and hence, caspase-3 activity was measured to investigate the impact of DZ on apoptotic events by ELISA. Caspase-3 activity in SCII rats was greatly increased compared with that in sham-operated rats $(\mathrm{P}<0.01$; Table II). Compared with that in the SCII group, DZ and MP treatment concomitantly attenuated caspase-3 activity $(\mathrm{P}<0.01)$.

Effect of DZ on apoptotic cells by TUNEL assay. TUNEL staining was performed to visualize apoptotic cells in spinal tissues of sham-operated control and experimental rats (Fig. 3). Histological sections of spines from SCII rats displayed an increased the number of TUNEL-positive cells in comparison with those of sham-operated control 

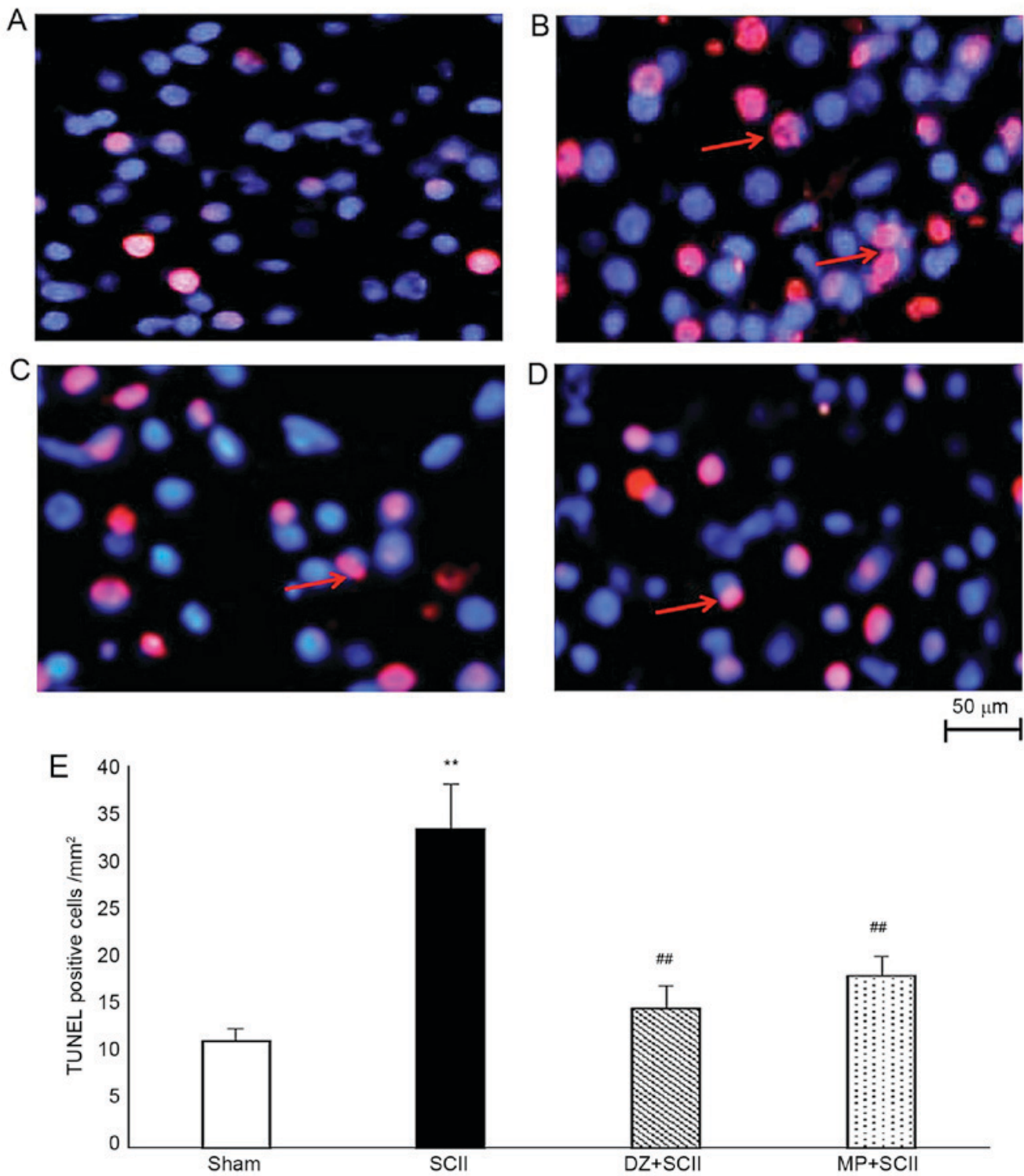

Figure 3. Effect of DZ on apoptosis in spinal sections of experimental rats as determined by TUNEL staining. (A) Sham-operated rats had only few TUNEL-positive cells, whereas (B) SCII-induced rats had an obviously increased number of TUNEL-positive cells (indicated by red arrows). However, in the (C) DZ and (D) MP groups, the number of TUNEL-positive cells (red) was decreased (scale bar, $50 \mu \mathrm{m}$ ). Nuclei were counter-stained with DAPI. (E) Number of TUNEL-positive cells was calculated. ${ }^{* *} \mathrm{P}<0.01$ vs. sham control group; ${ }^{\# t} \mathrm{P}<0.01$ vs. SCII group. DZ, daidzein; MP, methylprednisolone; TUNEL, terminal deoxynucleotidyl transferase-mediated deoxyuridine triphosphate nick-end labeling.

rats $(\mathrm{P}<0.01)$. However, the DZ and MP groups displayed a concomitant decline in the number of TUNEL-positive cells, compared with that in SCII rats $(\mathrm{P}<0.01)$.

Effect of DZ on the expression of PI3K/Akt and apoptotic signaling proteins. Western blot analysis was performed to examine the protein expression of pro-apoptotic protein Bax and anti-apoptotic protein Bcl-2 levels to determine the anti-apoptotic activity of DZ (Fig. 4). In addition, the protein levels of PI3K, Akt and pAkt were also determined to investigate whether this signaling pathway is associated with the neuroprotective properties of DZ (Fig. 5). The protein expression of $\mathrm{PI} 3 \mathrm{~K}, \mathrm{Bcl}-2$ and the pAkt/Akt ratio in the SCII group displayed a significant downregulation as compared with that in the sham-operated group $(\mathrm{P}<0.01)$, while the protein expression of Bax was markedly upregulated in SCII rats $(\mathrm{P}<0.01)$. However, compared with those in the
SCII group, the protein levels of PI3K, the pAkt/Akt ratio and Bcl-2 levels in the DZ and MP groups were significantly increased, while Bax levels were significantly downregulated $(\mathrm{P}<0.01$ or $<0.05)$.

\section{Discussion}

The present study was performed to assess the detailed molecular mechanisms of the neuroprotective effects of DZ in a rat model of SCII. The PI3K/Akt signaling pathway is crucial for the maintenance of cell survival (anti-apoptotic effect) and proliferation in most tissues, particularly neurons and glial cells $(17,19)$. Hence, the present study postulated that DZ probably exerts its neuroprotective effects by positively upregulating various downstream molecules of the PI3K/Akt signal transduction pathway. To prove this hypothesis, hind limb locomotor function of experimental rats was evaluated 

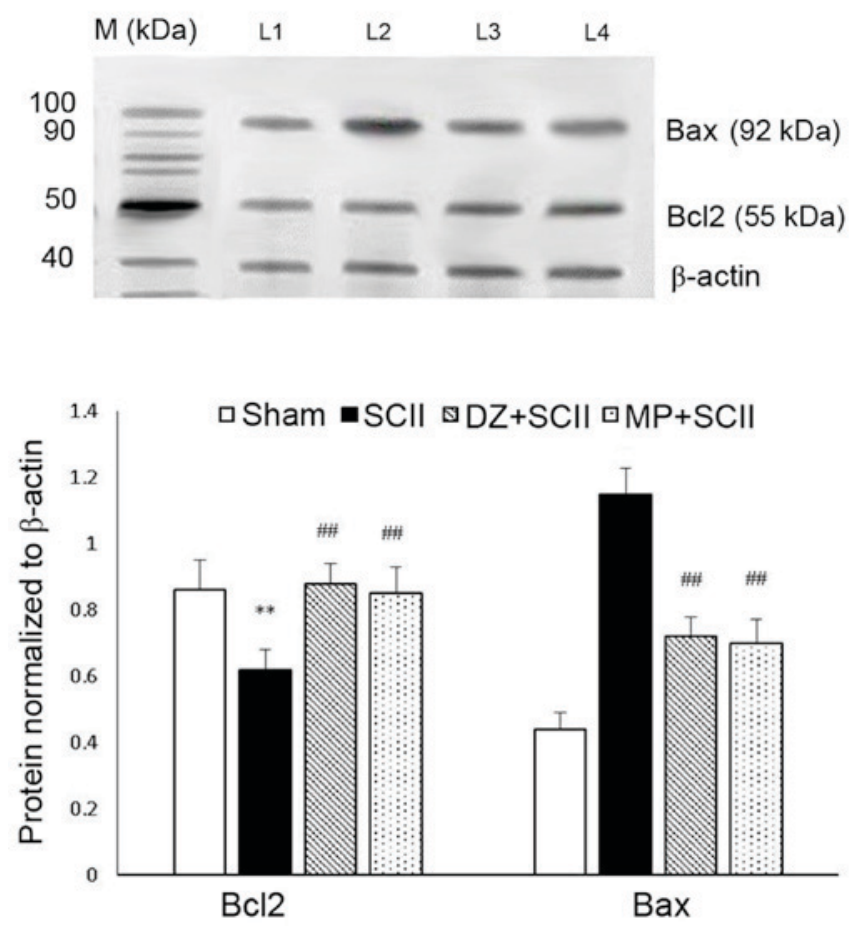

Figure 4. Effect of DZ on the protein expression of Bcl-2 and Bax in spinal tissue homogenate of experimental rats. Representative western blot images are displayed and quantitative values are expressed as the mean \pm standard deviation for twelve rats in each group. $\beta$-actin was used as internal standard Lanes: 1 , sham control group; 2 , SCII group; $3, \mathrm{DZ}+\mathrm{SCII}$ group; 4, MP+SCII group. ${ }^{* *} \mathrm{P}<0.01$ vs. sham control group; ${ }^{\# \#} \mathrm{P}<0.01$ vs. SCII group. DZ, daidzein; MP, methylprednisolone; SCII, spinal cord ischemia/reperfusion injury; Bcl-2, B-cell lymphoma 2; Bax, Bcl-2-associated X protein.

by BBB scoring, and oxidative stress (anti-oxidants), inflammatory markers (MPO, TNF- $\alpha$ and NF-p65), apoptotic markers (caspase-3, Bcl-2 and Bax) as well as the protein expression of signaling molecules such as PI3K and pAkt were assessed.

The neuroprotective efficacy of pharmaceutical drugs pertaining to the motor movement was screened through the BBB scoring system. It is one of the standard methods to measure the hind limb movement prior to and after injury $(7,20)$. The results of the present study indicated that SCII group rats showed a decreased BBB score due to the rapid onset of neurological damage owing to lack of sufficient blood circulation (hypoxia) under ischemic conditions. However, supplementation with DZ and MP significantly improved hind limb movement, which was reflected in a substantial increase in BBB score. Since DZ and MP act as a potent antioxidant and anti-inflammatory agents, they suppress oxidative stress and the subsequent inflammatory response to thereby lower neuronal damage or deficits. DZ has been reported to lower neurological deficits by improving the neuronal count and thus significantly ameliorating hind limb movement (10).

Spinal morphological abnormalities were circuitously analyzed via quantifying edema based on water content (dry and wet weight). SCI-induced rats displayed severe edematous conditions compared with sham-operated rats, likely due to ischemic/reperfusion causing electrolytic imbalance and resulting in a high-water content (edema). DZ- and

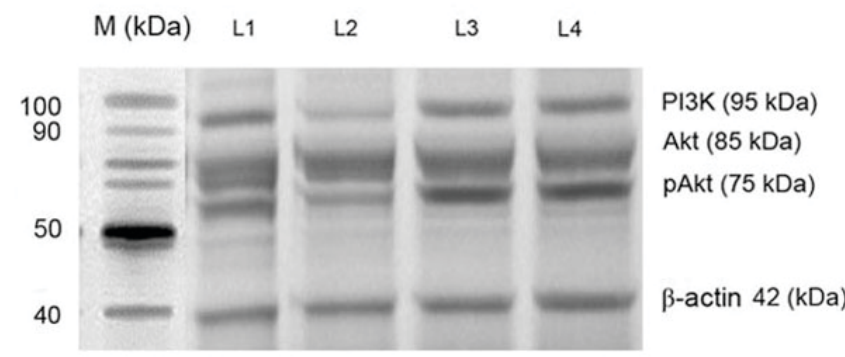

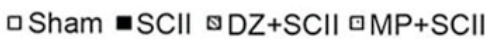

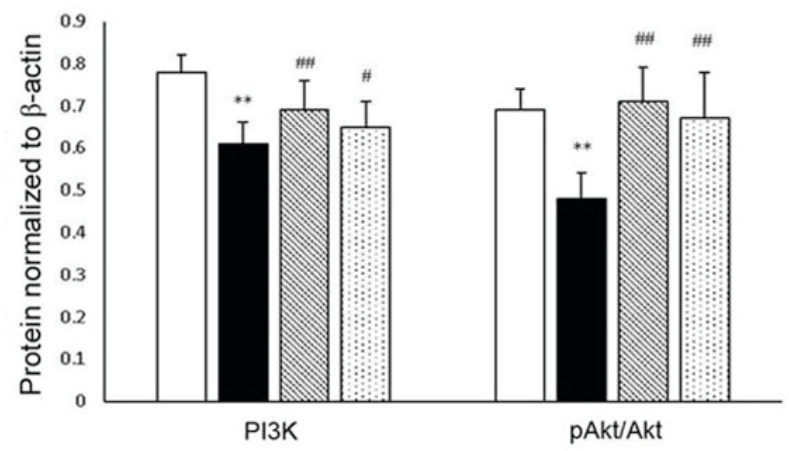

Figure 5. Effect of DZ on protein expression of PI3K and pAkt/Akt in spinal tissue homogenate of experimental rats. Representative western blot images are displayed and quantitative values are expressed as the mean \pm standard deviation for twelve rats in each group. $\beta$-actin was used as internal standard. Lanes: 1, sham control group; 2, SCII group; 3, DZ+SCII group; 4, MP+SCII group. ${ }^{* *} \mathrm{P}<0.01$ vs. sham control group; ${ }^{\# \#} \mathrm{P}<0.01,{ }^{\#} \mathrm{P}<0.05$ vs. SCII group. DZ daidzein; MP, methylprednisolone; SCII, spinal cord ischemia/reperfusion injury; pAkt, phosphorylated Akt; PI3K, phosphoinositide-3 kinase.

MP-treated rats demonstrated a decline in edema levels, probably through alteration of the electrolytic balance through maintenance of the blood-spinal cord barrier (BSCB). The results are in agreement with those of Ma et al (11), who reported that DZ treatment reduced edema in an ischemic rat model.

It is well documented that under ischemic conditions, free radicals are generated excessively as a cellular response to ischemic reperfusion, leading to oxidative stress (6). In the present study, SOD and CAT activity in SCII animals were significantly decreased owing to overproduction of free radicals (oxidative stress). The SCII-induced decreases in antioxidant activity (SOD and CAT) were reverted to near normal levels by administration of DZ and MP, likely via their antioxidant effects. Atkinson et al (22) demonstrated that DZ exhibits excellent antioxidant activity due to estrogenic activity. In addition, Xu et al (14) suggested that the isoflavone DZ may attenuate oxidative stress in a diabetic cell model owing to its two free hydroxyl groups. Zhang et al (23) proved that DZ activated the $\mathrm{Nrf} 2 /$ heme oxygenase-1 signaling pathway through the PI3K/Akt pathway. Hence, the present study hypothesized that DZ causes upregulation of various endogenous antioxidants, particularly SOD and CAT, via PI3K/Akt.

Oxidative stress and inflammation have been reported to be interlinked under spinal or cerebral ischemic/reperfusion conditions (20). Under SCII conditions, neutrophils and neuroglia are highly activated and mobilized (infiltration) 


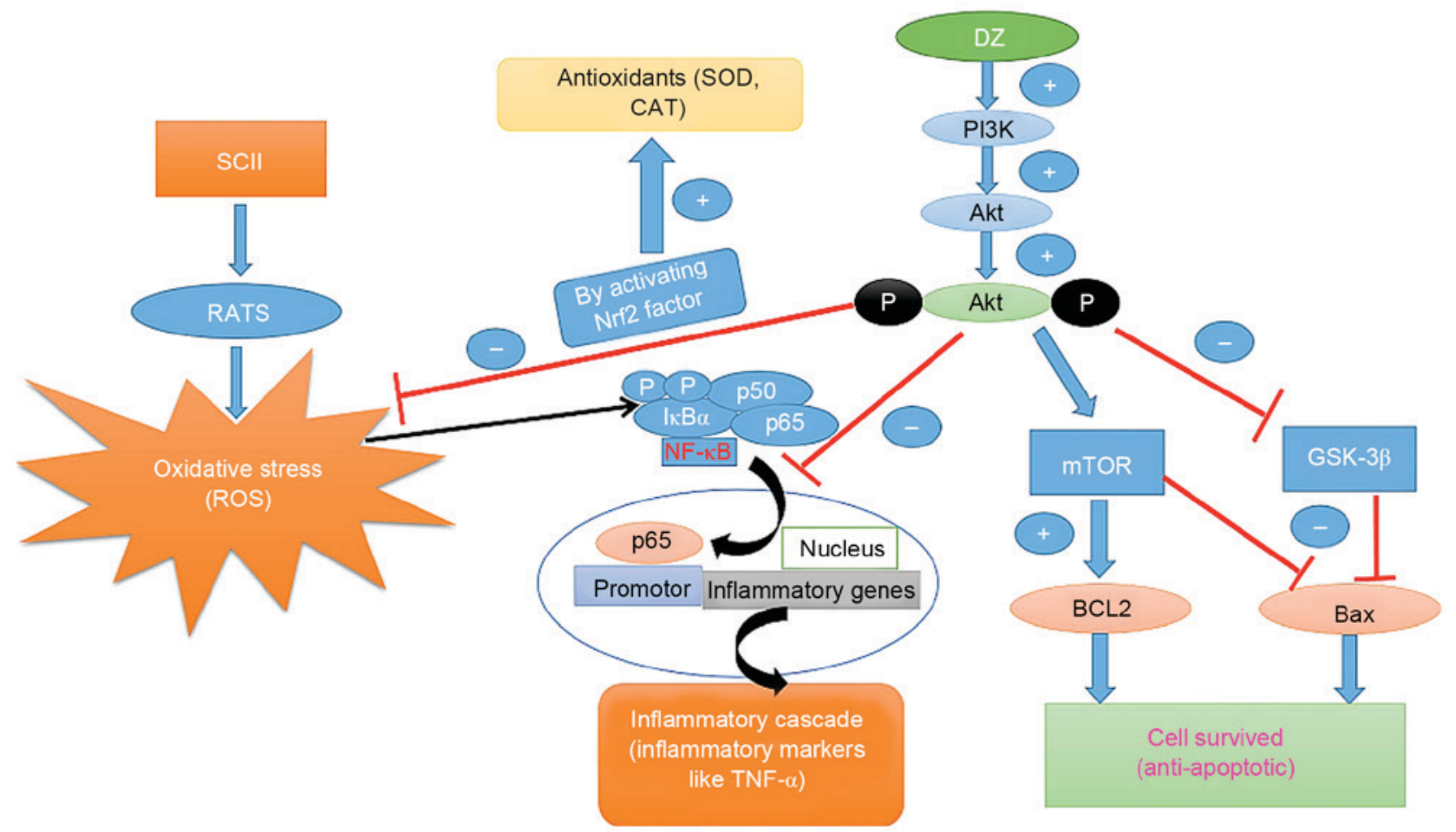

Figure 6. Schematic representing the in-depth molecular mechanism of the neuroprotective effect of DZ. DZ, daidzein; SCII, spinal cord ischemia/reperfusion

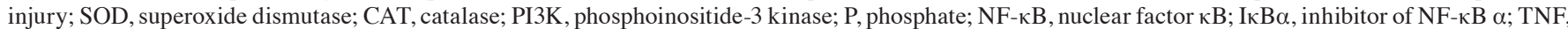
tumor necrosis factor; Bcl-2, B-cell lymphoma 2; Bax, Bcl-2-associated X protein; mTOR, mammalian target of rapamycin; GSK, glycogen synthase kinase; Nrf2, nuclear factor erythroid 2-related factor.

due to increased oxidative stress and disruption of BSCB. Those activated cells may initiate the inflammatory cascade by triggering $\mathrm{NF}-\mathrm{KB}$ and subsequently increasing various pro-inflammatory cytokines such as TNF- $\alpha$ (24). Hence, the present study initially measured the activity of MPO, which is the best marker for neutrophil infiltration, as well as various inflammatory markers, to determine the inflammatory status. The levels of MPO, TNF- $\alpha$ and NF- $\kappa \mathrm{B}$ p65 were elevated in SCII rats. However, DZ and MP substantially suppressed the levels of MPO, TNF- $\alpha$ and nuclear translocation of the NF- $\mathrm{KB}$ p65 subunit, thus indicating its anti-inflammatory activity by inactivating neutrophils and neuroglial cells. These results are congruent with those of Kim et al (25), who demonstrated that DZ administration reduced ischemia/reperfusion-induced myocardial damage in vivo via inhibition of $\mathrm{NF}-\mathrm{\kappa B}$ activation (nuclear translocation of NF- $\kappa \mathrm{B}$ p65).

Apoptosis is a programmed cell death, which is an integral part of various biological events. Apoptosis is upregulated in various neurodegenerative diseases, including Alzheimer's, Parkinson's and Huntington's disease as well as in traumatic injuries, particularly neurological injuries such as SCI $(26,27)$. The proliferative and apoptotic rate in each tissue type are maintained in a balance under normal conditions. Any alteration in either the proliferative or apoptotic rate may result in pathological conditions such as cancer, auto-immune diseases or degenerative diseases. Caspases (cysteine-aspartic proteases) are a family of proteases that has a critical role in executing apoptosis. Apoptotic caspases are classified into two types as the initiator (caspase- $2,-8$ and -9 ) and executor (caspase-3, -6 and -7) caspases. The initiator caspases activate executor caspases to trigger apoptosis (28). Among them, caspase- 3 is considered as the prime executioner protease of the apoptotic cascade through breaking DNA as well as disassembling its repair system (29). Therefore, the determination of caspase-3 levels as an apoptotic marker is a crucial criterion to assess apoptosis, particularly during post-ischemic conditions (6). In the present study, caspase-3 activity was significantly increased in SCI-insulted rats due to elevated oxidative stress and inflammatory response, which may hasten neuronal apoptosis under ischemic conditions. Compared with that in the SCII group, caspase-3 activity was concomitantly attenuated in the DZ- and MP-treated groups, probably as a result of the decreased oxidative stress and the inflammatory cascade. Rivera et al (9) indicated that supplementation with daidzein substantially reduced caspase-3 activity and thereby lowered apoptosis. Similarly, Aras et al (10) demonstrated that supplementation with daidzein substantially decreased the number of cells with positive caspase-3 staining by using immunohistochemistry.

Apoptosis is highly regulated by pro-apoptotic factors (Bax and Bcl-2-associated death promoter) and anti-apoptotic factors (Bcl-2 and $\mathrm{Bcl}$ extra large protein). Bax and Bcl-2 have an antagonising interaction (30), and in the present study, the expression levels of these two proteins we evaluated to determine apoptosis. The anti-apoptotic factor Bcl-2 usually resides in the mitochondrial outer membrane and in the nuclear membrane to prevent the release of cytochrome $C$ from mitochondria into the cytosol. Thus, Bcl-2 effectively inhibits caspase activation and thereby inactivates the triggering of the apoptotic caspase cascade. Furthermore, Bcl-2 inhibits the activation of pro-apoptotic factor Bax 
and thus potentially halts apoptotic events $(31,32)$. In the present study, the protein expression of Bcl-2 was markedly downregulated, whereas Bax was notably upregulated in the SCII group compared with that in the sham-operated group. Upon treatment with DZ and MP, the protein expression of Bcl-2 was markedly upregulated and Bax was substantially downregulated. Therefore, it was confirmed that DZ had effective anti-apoptotic activity by upregulating Bcl-2 and downregulating Bax to inactivate the apoptotic cascade. Mao et al (33) indicated that DZ acts as an anti-apoptotic agent by significantly downregulating the mRNA expression of the pro-apoptotic protein Bax and upregulating the anti-apoptotic protein $\mathrm{Bcl}-2$ in to inhibit D-galactose-induced neurotoxicity. In addition, DZ is an estrogen mimic with the ability to enhance cell proliferation and prevent neuronal loss (34). In line with this, the present study proved that DZ effectively attenuates caspase- 3 activity and hence inhibits apoptosis.

A TUNEL assay was performed to visualize the apoptotic cells, which were activated by pro-apoptotic proteins and the caspase cascade. The stained spinal sections of SCII rats had a significantly increased number of TUNEL-positive cells compared with those in the sham group. However, sections of DZ- or MP-treated rats had a significantly decreased number of TUNEL-positive cells compared with those in SCII rats. In a previous study, the DZ metabolite equol exerted significant anti-apoptotic activity as reflected by a decreased number of TUNEL-positive cells in a $\mathrm{H}_{2} \mathrm{O}_{2}$-induced endothelial cell cytotoxicity model (23). The present study proved that DZ substantially decreased apoptosis by altering the expression of pro- and anti-apoptotic proteins and thereby attenuating the concentration of caspase-3.

As mentioned previously, PI3K/Akt pathway has a pivotal role in neuronal regeneration after acute SCII (19). In addition, numerous studies have indicated that the neuroprotective activity of soy beans is mainly bestowed by DZ, geinstein and equol (metabolite of DZ) probably due to their estrogenic activity via the PI3K/Akt pathway $(11,35)$. Hence, the present study assessed whether DZ exerts its neuroprotective activity by upregulating PI3K/Akt signaling molecules to lower apoptosis and enhance regeneration of neurons. The protein expression and the pAkt/Akt ratio were significantly decreased in ischemic/reperfusion-induced animals, which was inhibited by intervention with DZ. Since DZ is an estrogen mimic, it activates PI3K/Akt-dependent downstream molecules, which may be a key mechanism underlying its neuroprotective properties (35,36). Zhang et al (23) demonstrated that S-equol exerts its neuroprotective function via activation of $\mathrm{Nrf} 2$ through $\mathrm{Akt}$ phosphorylation. A previous study also demonstrated that DZ downregulated GSK-3 $\beta$ and upregulated mTOR and thereby inhibited apoptosis through the activation of Bcl-2 in an ischemic model (37). Fig. 6 presents a schematic of the in-depth molecular mechanism of the neuroprotective effect of DZ identified in the present study.

Of note, the present study had certain limitations. For instance, the BBB staining assay to assess the integrity of neurons (tight junctions), and detection of markers for mitochondrial dysfunction (membrane potential) was not performed. Furthermore, the levels of GSK-3 $\beta$ and mTOR were not measured to confirm the anti-apoptotic activity of DZ.
In conclusion, the present study suggested that pre- and post-SCII treatment with DZ for seven consecutive days each considerably inhibited impairments of hind limb movement (motor function) via activation of PI3K and Akt, likely through inhibiting GSK-3 $\beta$ as well as activation (phosphorylation) of mTOR. Activated mTOR activates anti-apoptotic Bcl-2 protein, which in turn inhibits pro-apoptotic Bax protein as well as inactivates caspase-3 enzyme to promote cell survival/proliferation and thereby maintain/restore neuronal integrity and motor function. Thus, DZ restrained apoptosis through the PI3K/Akt signaling pathway to exert its neuroprotective activity. Further study is required to elucidate the complete mechanism of the neuroprotective effects of DZ.

\section{References}

1. Wong DR, Coselli JS, Amerman K, Bozinovski J, Carter SA, Vaughn WK and LeMaire SA: Delayed spinal cord deficits after thoracoabdominal aortic aneurysm repair. Ann Thorac Surg 83: 1345-1355, 2007.

2. Kanellopoulos GK, Xu XM, Hsu CY, Lu X, Sundt TM and Kouchoukos NT: White matter injury in spinal cord ischemia protection by AMPA/kainate glutamate receptor antagonism. Stroke 31: 1945-1952, 2000.

3. Zhang P, Zhang L, Zhu L, Chen F, Zhou S, Tian T, Zhang Y, Jiang X, $\mathrm{Li}$ X, Zhang C and Xu L: The change tendency of PI3K/Akt pathway after spinal cord injury. Am J Transl Res 7: 2223-2232, 2015.

4. Maniar HS, Sundt TM III, Prasad SM, Chu CM, Camillo CJ, Moon MR, Rubin BG and Sicard GA: Delayed paraplegia after thoracic and thoracoabdominal aneurysm repair: A continuing risk. Ann Thorac Surg 75: 113-119, 2003.

5. Wang Z, Zhang $\mathrm{C}$, Hong Z, Chen $\mathrm{H}$, Chen $\mathrm{W}$ and Chen $\mathrm{G}$ : C/EBP homologous protein (CHOP) mediates neuronal apoptosis in rats with spinal cord injury. Exp Ther Med 5: 107-111, 2013.

6. Gokce EC, Kahveci R, Gokce A, Sargon MF, Kisa U, Aksoy N, Cemil B and Erdogan B: Curcumin attenuates inflammation, oxidative stress, and ultrastructural damage induced by spinal cord ischemia-reperfusion injury in rats. J Stroke Cerebrovasc Dis 25: 1196-1207, 2016.

7. Lafci G, Gedik HS, Korkmaz K, Erdem H, Cicek OF, Nacar OA, Yildirim L, Kaya E and Ankarali H: Efficacy of iloprost and montelukast combination on spinal cord ischemia/reperfusion injury in a rat model. J Cardiothorac Surg 8: 64, 2013.

8. Burguete MC, Torregrosa G, Pérez-Asensio FJ, Castelló-Ruiz M, Salom JB, Gil JV and Alborch E: Dietary phytoestrogens improve stroke outcome after transient focal cerebral ischemia in rats. Eur J Neurosci 23: 703-710, 2006.

9. Rivera P, Pérez-Martín M, Pavón FJ, Serrano A, Crespillo A, Cifuentes M, López-Ávalos MD, Grondona JM, Vida M and Fernández-Llebrez P: Pharmacological administration of the isoflavone daidzein enhances cell proliferation and reduces high fat diet-induced apoptosis and gliosis in the rat hippocampus. PLoS One 8: e64750, 2013.

10. Aras AB, Guven M, Akman T, Ozkan A, Sen HM, Duz U, Kalkan Y, Silan C and Cosar M: Neuroprotective effects of daidzein on focal cerebral ischemia injury in rats. Neural Regener Res 10: 146-152, 2015.

11. Ma Y, Sullivan JC and Schreihofer DA: Dietary genistein and equol (4',7 isoflavandiol) reduce oxidative stress and protect rats against focal cerebral ischemia. Am J Physiol Regul Integr Comp Physiol 299: R871-R877, 2010.

12. Wu J, Oka J, Ezaki J, Ohtomo T, Ueno T, Uchiyama S, Toda T, Uehara $\mathrm{M}$ and Ishimi Y: Possible role of equol status in the effects of isoflavone on bone and fat mass in postmenopausal Japanese women: A double-blind, randomized, controlled trial. Menopause 14: 866-874, 2007.

13. Prossnitz ER and Barton M: Estrogen biology: New insights into GPER function and clinical opportunities. Mol Cell Endocrinol 389: 71-83, 2014.

14. Xu SZ, Zhong W, Ghavideldarestani M, Saurabh R, Lindow SW and Atkin SL: Multiple mechanisms of soy isoflavones against oxidative stress-induced endothelium injury. Free Radic Biol Med 47: 167-175, 2009. 
15. Lovekamp-Swan T, Glendenning $M$ and Schreihofer DA: A high soy diet reduces programmed cell death and enhances bcl-xl expression in experimental stroke. Neuroscience 148 644-652, 2007.

16. Pettiford JN, Bikhchandani J, Ostlie DJ, St Peter SD, Sharp RJ and Juang D: A review: The role of high dose methylprednisolone in spinal cord trauma in children. Pediatr Surg Int 28: 287-294, 2012

17. Zhang J, Deng Z, Liao J, Song C, Liang C, Xue H, Wang L, Zhang $\mathrm{K}$ and Yan $\mathrm{G}$ : Leptin attenuates cerebral ischemia injury through the promotion of energy metabolism via the PI3K/Akt pathway. J Cereb Blood Flow Metab 33: 567-574, 2013.

18. Zhang P, Zhang L, Zhu L, Chen F, Zhou S, Tian T, Zhang Y, Jiang X, Li X and Zhang C: The change tendency of PI3K/Akt pathway after spinal cord injury. Am J Transl Res 7: 2223-2232, 2015.

19. Jung SY, Kim DY, Yune TY, Shin DH, Baek SB and Kim CJ: Treadmill exercise reduces spinal cord injury-induced apoptosis by activating the PI3K/Akt pathway in rats. Exp Ther Med 7: 587-593, 2014.

20. Hwang JY, Min SW, Jeon YT, Hwang JW, Park SH, Kim JH and Han SH: Effect of coenzyme Q10 on spinal cord ischemia-reperfusion injury. J Neurosurg Spine 22: 432-438, 2015.

21. Mdzinarishvili A, Kiewert C, Kumar V, Hillert M and Klein J: Bilobalide prevents ischemia-induced edema formation in vitro and in vivo. Neuroscience 144: 217-222, 2007.

22. Atkinson C, Frankenfeld CL and Lampe JW: Gut bacterial metabolism of the soy isoflavone daidzein: Exploring the relevance to human health. Exp Biol Med 230: 155-170, 2005.

23. Zhang T, Liang X, Shi L, Wang L, Chen J, Kang C, Zhu J and Mi M: Estrogen receptor and PI3K/Akt signaling pathway involvement in S-(-)equol-induced activation of Nrf2/ARE in endothelial cells. PLoS One 8: e79075, 2013.

24. Palencia G, Medrano JÁ, Ortiz-Plata A, Farfán DJ, Sotelo J, Sánchez A and Trejo-Solís C: Anti-apoptotic, anti-oxidant and anti-inflammatory effects of thalidomide on cerebral ischemia/reperfusion injury in rats. J Neurol Sci 351: 78-87, 2015.

25. Kim JW, Jin YC, Kim YM, Rhie S, Kim HJ, Seo HG, Lee JH, Ha YL and Chang KC: Daidzein administration in vivo reduces myocardial injury in a rat ischemia/reperfusion model by inhibiting NF-kappaB activation. Life Sci 84: 227-234, 2009.

26. Hwang L, Choi IY, Kim SE, Ko IG, Shin MS, Kim CJ, Kim SH, Jin JJ, Chung JY and Yi JW: Dexmedetomidine ameliorates intracerebral hemorrhage-induced memory impairment by inhibiting apoptosis and enhancing brain-derived neurotrophic factor expression in the rat hippocampus. Int J Mol Med 31 1047-1056, 2013.
27. Sung YH, Kim SC, Hong HP, Park CY, Shin MS, Kim CJ, Seo JH, Kim DY, Kim DJ and Cho HJ: Treadmill exercise ameliorates dopaminergic neuronal loss through suppressing microglial activation in Parkinson's disease mice. Life Sci 91: 1309-1316, 2012

28. Riedl SJ and Shi Y: Molecular mechanisms of caspase regulation during apoptosis. Nat Rev Mol Cell Biol 5: 897-907, 2004.

29. Rami A: Ischemic neuronal death in the rat hippocampus: The calpain-calpastatin-caspase hypothesis. Neurobiol Dis 13: $75-88,2003$.

30. Okuno S, Saito A, Hayashi T and Chan PH: The c-Jun $\mathrm{N}$-terminal protein kinase signaling pathway mediates Bax activation and subsequent neuronal apoptosis through interaction with Bim after transient focal cerebral ischemia. J Neurosci 24: 7879-7887, 2004.

31. Chen MH, Ren QX, Yang WF, Chen XL, Lu C and Sun J: Influences of HIF-la on Bax/Bcl-2 and VEGF expressions in rats with spinal cord injury. Int J Clin Exp Pathol 6: 2312-2322, 2013.

32. Li Y, Gu J, Liu Y, Long H, Wang G, Yin G and Fan J: iNOS participates in apoptosis of spinal cord neurons via p-BAD dephosphorylation following ischemia/reperfusion (I/R) injury in rat spinal cord. Neurosci Lett 545: 117-122, 2013.

33. Mao Z, Zheng YL, Zhang YQ, Han BP, Zhu XW, Chang Q and $\mathrm{Hu}$ XB: The anti-apoptosis effects of daidzein in the brain of D-galactose treated mice. Molecules 12: 1455-1470, 2007.

34. Han BJ, Li W, Jiang GB, Lai SH, Zhang C, Zeng CC and Liu YJ: Effects of daidzein in regards to cytotoxicity in vitro, apoptosis, reactive oxygen species level, cell cycle arrest and the expression of caspase and Bcl-2 family proteins. Oncol Rep 34: 1115-1120, 2015.

35. Chakrabarti M, Das A, Samantaray S, Smith JA, Banik NL, Haque A and Ray SK: Molecular mechanisms of estrogen for neuroprotection in spinal cord injury and traumatic brain injury. Rev Neurosci 27: 271-281, 2016.

36. Yu F, Sugawara T, Maier CM, Hsieh LB and Chan PH: Akt/Bad signaling and motor neuron survival after spinal cord injury. Neurobiol Dis 20: 491-499, 2005.

37. Kim MH, Park JS, Seo MS, Jung JW, Lee YS and Kang KS: Genistein and daidzein repress adipogenic differentiation of human adipose tissue-derived mesenchymal stem cells via Wnt/ $\beta$-catenin signalling or lipolysis. Cell Prolif 43: 594-605, 2010 . 